\title{
External Quality Assessment of Laboratories Performance in AFB Microscopy in Tigray Region, North Ethiopia 2016
}

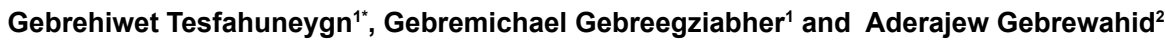

${ }^{1}$ Tigray Health Research Institute, Mekelle, Ethiopia

${ }^{2}$ Adigrat University, Adigrat, Ethiopia

\begin{abstract}
Background: In several countries with a high incidence of tuberculosis (TB), direct sputum smear microscopy remains the most cost effective tool for diagnosing patients with infectious tuberculosis and for monitoring their progress on treatment. The objective of the current study was to assess the quality of laboratory service in AFB microscopy of Tigray Region. Randomly selected health facilities were participated in the external quality assessment. Data was entered and analyzed using SPSS version 20.
\end{abstract}

Results: Between December 2015 and February 2016, a total of 80 laboratories were participated. Most (87\%) of the laboratories have continuous electric supply. Only $70 \%$ of the health facilities have continuous pipe water and $56.5 \%$ have no separate table for smear preparation, staining, microscopy and recording. From the distributed panel slides, $91 \%$ of the Health facilities were scored $100 \%$ concordant results but $9 \%$ of the health facilities were scored discordant results. The main reasons for discordant results were shortage of training, nonfunctional microscope, have no lens cleaning and using of unfiltered carbol fuchsin reagent.

Conclusions: The performance of health facilities for smear microscopy reading and smear quality were relatively low in this study area, because smear positivity rate and annual negative slide volume of the health facilities were below the standard. Therefore Strengthening the EQA program and technical support on smear quality indicators are recommended to ensure for better quality diagnostic service of tuberculosis.

Keywords: Tuberculosis; Multi-drug resistant; Infection

\section{Introduction}

In several countries with a high incidence of tuberculosis (TB), direct sputum smear microscopy remains the most cost effective tool for diagnosing patients with infectious tuberculosis and for monitoring their progress on treatment. The current increase in multi-drug resistant TB (MDR-TB) cases and the emergence of extensive drug resistant TB (XDR-TB) refers that TB laboratory services should be performed totally according to the appropriate standards. Delayed both diagnosis of TB and determination of drug resistance have contributed to the dissemination of MDR- and XDR-TB. The main purpose in tuberculosis laboratory is to generate accurate, reliable and timely test results for clinicians [1-3].

The World Health Organization strategy for tuberculosis control (DOTS) depends on a network of laboratories that provide acid fast bacilli (AFB) sputum smear microscopy. The establishment of a broad network of well-functioning peripheral laboratories within the context of the health system and readily accessible to the population is a high priority for any tuberculosis control program [4]. If the laboratory diagnosis is unreliable, all other activities will be affected. However, the quality of laboratory services often may not be considered a high priority of the National Tuberculosis Program (NTP). Microscopy errors are likely to result in failure to detect persons with infectious TB who will then continue to spread infection in the community or unnecessary treatment for "noncases." Errors in reading follow up smears can result in patients being placed on prolonged treatment, retreatment and in treatment discontinued prematurely [2]. Therefore, quality assurance of laboratory services, including AFB sputum smear microscopy, is essential. Both the availability and quality of AFB smear microscopy are dependent on national programs that support, train and monitor the testing performance of individual laboratories. The current study was aimed to assess the quality of laboratory service in AFB microscopy in Tigray Region.

\section{Materials and Methods}

\section{Study design, period and area}

The State of Tigray has an estimated area of 80,000 square kilometers. According to the 2007 Census the state's population size was $4,316,988$ of which 2, 126, 465 were males and 2, 190, 523 were females. The urban residents of the region number 844,040 and its rural residents $3,472,948$. The region has 102 ART centers. All of these health institutions send sample for laboratory investigation to Tigary health research institute. A cross sectional study was conducted between December 2015 and February 2016.

\section{Study subjects, sample size and sampling method}

Randomly selected health facilities were participated in the external quality assessment. Structured checklists and panel test slides were prepared. Data was collected using structured checklists and standard formats for recording results of panel test slides.

\section{Data collection procedure and quality control}

Data were collected using the three external quality assessment methods (on site evaluation, blinded rechecking and panel tests).

\section{Onsite evaluation}

This was conducted using the standard checklist to assess the three phases of the Laboratory work flow and overall working condition.

*Corresponding author: Gebrehiwet Tesfahuneygn, Tigray Health Research Institute, Mekelle, Ethiopia, Tel, +251-342414330, E-mail: gtlab2006@gmail.com

Received September 04, 2017; Accepted November 21, 2017; Published November 29, 2017

Citation: Tesfahuneygn G, Gebreegziabher G, Gebrewahid A (2017) External Quality Assessment of Laboratories Performance in AFB Microscopy in Tigray Region, North Ethiopia 2016. Lung Dis Treat 3: 126. doi:10.4172/2472-1018.1000126

Copyright: @ 2017 Tesfahuneygn G, et al. This is an open-access article distributed under the terms of the Creative Commons Attribution License, which permits unrestricted use, distribution, and reproduction in any medium, provided the original author and source are credited. 


\section{Blinded rechecking}

Health facilities stored slide sequentially based on national guideline. Sample size (volume of slides) was based on modified statistical sampling method called Lot Quality assurance sampling (LQAS). This depends on the negative slide volume and slide positivity rate of the previous year.

\section{Panel testing}

For the panel testing, positive and negative sputum samples were collected and processed in Tigray health research institute. Negative smears and different grades of positive smears were prepared for panel testing. Samples were taken from each negative and positive prepared slide. The slides were stained by $\mathrm{ZN}$ staining technique to check for quality of smears and grades of positive slides using $100 \times$ objectives. Unstained panel test slides were then dispatched to the peripheral laboratories. A total of 800 panel test slides with different results were prepared and 10 slides from each were distributed to each peripheral laboratory and pass mark was determined based on national guidelines. All the laboratory procedures were conducted following the standard operating procedure (SOP). The quality of data was checked by reviewing the questionnaire for consistency and extensiveness.

\section{Data processing and analysis}

Data were entered, cleaned and analyzed using SPSS version 20. Smear quality indicators and AFB results were calculated. Each laboratory was evaluated for major errors indicate gross technical deficiencies and include both high false positive (HFP) and high false negative (HFN) errors. It was considered as HFP when a negative smear was misread as $1+$ to $3+$ and HFN when a $1+$ to $3+$ positive smear was misread as negative. Minor errors indicate low false positive (LFP), low false negative (LFN) and quantification error (QE). It was considered as LFP when a negative smear was misread as low positive (1-9AFB/100 fields) and LFN when a low positive smear (1-9AFB/100 fields) was misread as negative. Variables having $\mathrm{P}$ value $<0.05$ in bivariate analysis were entered into multivariate analysis to manage confounder variables. Significant association was set at $\mathrm{P}$ value $<0.05$.

\section{Ethical approval}

Ethical approval was obtained from Tigray regional health bureau and permission was obtained from each health facilities before conducting this study.

\section{Results}

A total of 80 laboratories were participated. Most $(87 \%)$ of the laboratories have continuous electric supply. Only $70 \%$ of the health facilities have continuous pipe water and $56.5 \%$ have no separate table for smear preparation, staining, microscopy and recording. Most laboratories decontaminate sputum before disposal (Table 1).

\begin{tabular}{|c|c|c|}
\hline Characteristics & Yes n (\%) & No n (\%) \\
\hline Separate table/ area for TB & $37(46)$ & $43(54)$ \\
\hline Separate microscope for TB & $18(22.5)$ & $62(77.5)$ \\
\hline Running water in the laboratory & $56(70)$ & $24(30)$ \\
\hline Preventive maintenance & $70(87.5)$ & $10(12.5)$ \\
\hline Participated in EQA program & $80(100)$ & $0(0)$ \\
\hline Received EQA feedback & $52(65)$ & $28(35)$ \\
\hline National guideline & $80(100)$ & $0(00$ \\
\hline Using of positive and negative slides for QC & $56(70)$ & $24(30)$ \\
\hline Availability of job aids & $76(95)$ & $4(5)$ \\
\hline
\end{tabular}

Table 1: General characteristics of laboratories in Tigray region, between December 2015 and February 2016.

\section{On site evaluation}

In fourteen percent laboratories have shortage of AFB reagents and only $71 \%$ of laboratories performed internal quality control (IQC) for new AFB reagents and routinely in a weekly manner using known positive and negative control smears. Ten percent laboratories used AFB reagents with expired reagent and with no concentration label. $24 \%$ laboratories didn't filter AFB reagents and 29\% laboratories didn't' use tissue paper to clean microscope due to stock out.

\section{Annual negative slide volume and slide positivity rate}

Standard request paper for AFB is not available in $29 \%$ laboratories and $10 \%$ laboratories didn't adhere to the national guideline grading system to report client results. Majority of the health facilities have low annual slide number $(<300 /$ year $)$ and slide positivity rate. The average slide positivity rate of health facilities was $6.9 \%$ (Table 2 ).

\section{Panel test}

$800 \mathrm{AFB}$ panel slides prepared of which 480 positive with different grades and 320 negative. We had distributed to each health facilities according to the national guideline and $91 \%$ of the health facilities were scored $100 \%$ concordant results but $9 \%$ of the health facilities were scored discordant results. The main reasons for discordant results were Shortage of training, nonfunctional microscope, have no lens cleaning and using of unfiltered carbol fuchsin reagent (Figure 1).

\section{Blinded rechecking}

A total of 3246 slides were collected from the health facilities using EQA guideline. Among the collected slides 129 (4\%) were reported positive and 3117 (96\%) negative at the peripheral laboratories. Most (98\%) of the slides were concordant results but in $2 \%$ health facilities discordant results were reported. The quality of most (80\%) slides was normal regarding the six quality indicators.

\section{Discussion}

Well-timed and accurate diagnosis of TB and proper treatment are essential for dropping disease burden as well as transmission in

\begin{tabular}{|c|c|c|c|c|}
\hline ANSV & $\begin{array}{c}\text { SPR<5 } \\
\mathbf{N}(\%)\end{array}$ & $\begin{array}{c}\text { SPR5-10 } \\
\mathbf{N}(\%)\end{array}$ & $\begin{array}{c}\text { SPR>10 } \\
\mathbf{N}(\%)\end{array}$ & $\begin{array}{c}\text { Total } \\
\mathbf{N}(\%)\end{array}$ \\
\hline$<300$ & $20(25)$ & $12(15)$ & $7(8.75)$ & $39(48.75)$ \\
\hline $301-500$ & $12(15)$ & $4(5)$ & $11(13.75)$ & $27(33.75)$ \\
\hline $501-1000$ & $0(0)$ & $7(8.75)$ & $0(0)$ & $7(8.75)$ \\
\hline$>1000$ & $0(0)$ & $7(8.75)$ & $0(0)$ & $7(8.75)$ \\
\hline Total & $32(40)$ & $30(37.5)$ & $18(22.5)$ & $80(100)$ \\
\hline
\end{tabular}

2: ANSV and SPR of smear microscopy in Tigray region between 2015 and February 2016.

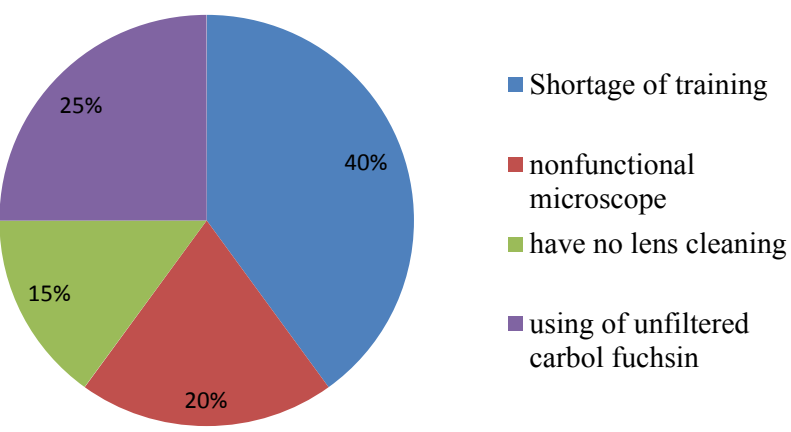

Figure 1: Main reasons for poor TB performance. 
the community [5]. Even though quality assurance intuberculosis laboratories is an indispensable component of effective tuberculosis control, quality assurance in the absence of an effective treatment program will have little impact and is a misdirected priority. Thus, a well-functioning national TB control program, including case finding by sputum smear microscopy and the provision of effective treatment based on the DOTS strategy, is an absolute precondition to a fruitful Quality Assurance Program [6].

In this study the average panel test score of the participating laboratories were $91 \%$. This is relatively high compared to other studies conducted in Ethiopia, Amahara region which was 77\% [7]. This difference may due method difference because we used three methods (blind rechecking, proficiency testing and onsite evaluation) but they used only blind rechecking. In our finding false positive results was reported in $4.7 \%$ laboratories. This is lower than study conducted in Ethiopia Amhara region which was 14.4\% [7]. But higher than studies conducted inTanzania, India and Iraq that reported no false positive result [8-10]. Laboratory false Positive smear results leads to patient receiving unnecessary treatment with toxic drugs and in addition puts precious resources of the programme to drain, increasing the health care costs [11].

In this study reported that SPR $<5 \%$ was $40 \%$. This is different from study conducted in Amhara region, Ethiopia which was 83.1\% [7]. This difference may be due to geographical difference. In our set up there is continuous assessment and training based on their gaps. Both low SPR and ANSV were found in $23 \%$ of laboratories. This finding is higher compared to the $2.9 \%$ of diagnostic centers with low SPR $(<5 \%)$ and low ANSV ( $<301$ slides) reported in New Delhi [12]. In contrast it is lower than study conducted in Ethiopia which was 39.8\% [7].

This study demonstrates false negativity rate was $9 \%$. The finding is different from studies done in Ethiopia, Vietnam, India and Iraq that reported the $20.4 \%, 18.7 \%, 3.7 \%$ and $2.3 \%$ of false negative results, respectively $[5,12,13]$. False negative results may be due to nonfunctional microscope, knowledge gap, reagent quality and not scanning of 100 fields before reporting client results.

\section{Conclusion}

In conclusion the performance of health facilities for smear microscopy reading and smear quality were relatively low in this study area, because smear positivity rate and ANSV of the health facilities were below the standard. In addition the infrastructure of TB laboratory is also poor. Therefore strengthening the EQA program and technical support on smear quality indicators are recommended to ensure for better quality diagnostic service of tuberculosis.

\section{Competing Interests}

The authors declare that they have no competing interests.

\section{Authors' Contribution}

GT, GG and AG designed the study, participated in data collection, analysis, interpretation and write-up, drafted the manuscript and critically revised the manuscript. All authors read and approved the final manuscript.

\section{Acknowledgements}

We would like to acknowledge Tigray health bureau, THRI and we also like to thanks to each health facility heads and laboratories staff.

\section{Reference}

1. Zignol M, Gemert W, Falzon D, Jaramillo E, Blanc L, et al. (2011) Modernizing surveillance of antituberculosis drug resistance: From special surveys to routine testing. Clin Infect Dis 52: 901-906.

2. Pai M, Daley P, Hopewell PC (2007). International standards for tuberculosiscare: Relevance and implications for laboratory professionals. Indian J Med Microbiol 25: 89-92.

3. Ozkutuk A (2009) Quality Assurance in Tuberculosis Laboratories. Mikrobiyol Bul 43: 699-707.

4. International Organization for Standardization ISO/TC 212/WG (19961) Quality Management in the Clinical Laboratory. ISO/IEC Guide 43, Proficiency Testing by Interlaboratory Comparisons.

5. Paramasivan $\mathrm{C}$,Venkataraman $\mathrm{N}$, Vasanthan $\mathrm{P}$, Rahman $\mathrm{J}$, Narayanan $\mathrm{F}$ (2003) Quality assurance studies in eight state tuberculosis laboratories in India. Int J Tuberc Lung Dis 7: 522-527.

6. Martinez-Guarneros A, Balandrano-Campos S, Solano-Ceh MA, GonzalezDominguez F, Lipman HB, et al. (2002) Implementation of proficiency testing in conjunction with a rechecking system for external quality assurance in tuberculosis laboratories in Mexico 7: 516-521.

7. Shiferaw MB, Hailu HA, Fola AA, Derebe MM, Kebede AT, et al. (2015) Tuberculosis Laboratory Diagnosis Quality Assurance among Public Health Facilities in West AmharaRegion, Ethiopia. PLoS ONE 10: e0138488.

8. Basra D, Matee M, Mcnerney R (2006) Quality assessment of sputum smears microscopy for detection of Acid fast bacilli in peripheral health care facilities in Dar Es Salaam, Tanzania. East Afr Med J 83: 306-310.

9. Sarin R, Singla N, Mukerjee S, Sharma P (2002) Quality control of sputum microscopy at sub-district level. Ind J Tub 49:143.

10. Farnia P, Masjedi M, Mohammadi F, Nasehi M, Foroozesh M, et al. (2003) The results of three years surveillance on sputum smear microscopy in 285 district and regional tuberculosis laboratories of Iran. Tanaffos 2: 29-36.

11. http://medind.nic.in/nac/t05/i1/nact05i1p18.pd

12. Rie A, Fitzgerald D, Kabuya G, Deun A, Tabala M, et al. (2008) Sputum smea microscopy: Evaluationof impact of training, microscope distribution, and use of external quality assessment guidelines for resource-poor settings. J ClinMicrobiol 46: 897-901.

13. Nguyen TN, Wells CD, Binkin NJ, Becerra JE, Pham DL, et al. (1999) Quality control of smear microscopy for acid-fast bacilli: The case for blinded rereading. Int J Tuberc Lung Dis 3: 55-61. 